\title{
High speed multiphoton imaging
}

\author{
Yongxiao Li ${ }^{\mathrm{a}}$, Anne Brustle ${ }^{\mathrm{b}}$, Vini Gautamº ${ }^{\mathrm{b}}$ Ian Cockburn ${ }^{\mathrm{b}}$, Cathy Gillespie ${ }^{\mathrm{b}}$, Katharina Gaus ${ }^{\mathrm{c}}$, \\ Woei Ming Lee*a \\ ${ }^{a}$ Research School of Engineering, College of Engineering and Computer Science, The Australian \\ National University, 31 North Road, Canberra, ACT, Australia 2601; bJonh Curtin School of \\ Medical Research, The Australian National University, Canberra, ACT, Australia 2600; 'Australia- \\ EMBL Single Molecule Science, University of New South Wales, Sydney, NSW, Australia 2052.
}

\begin{abstract}
Intravital multiphoton microscopy has emerged as a powerful technique to visualize cellular processes in-vivo. Real time processes revealed through live imaging provided many opportunities to capture cellular activities in living animals. The typical parameters that determine the performance of multiphoton microscopy are speed, field of view, 3D imaging and imaging depth; many of these are important to achieving data from in-vivo. Here, we provide a full exposition of the flexible polygon mirror based high speed laser scanning multiphoton imaging system, PCI-6110 card (National Instruments) and high speed analog frame grabber card (Matrox Solios eA/XA), which allows for rapid adjustments between frame rates i.e. $5 \mathrm{~Hz}$ to $50 \mathrm{~Hz}$ with 512 x 512 pixels. Furthermore, a motion correction algorithm is also used to mitigate motion artifacts. A customized control software called Pscan 1.0 is developed for the system. This is then followed by calibration of the imaging performance of the system and a series of quantitative in-vitro and in-vivo imaging in neuronal tissues and mice.
\end{abstract}

Keywords: Multiphoton microscopy, high speed scanning, polygon mirror, customized control software

\section{INTRODUCTION}

Since first invented by Denk in 1990, two photon microscope received a lot of attentions from biologists, especially for neuroscience and in-vivo calcium imaging ${ }^{1}$. The feature of low photon bleaching and photon toxicity as well as the deep imaging ability, since the two photon absorption law, makes two photon microscope an ideal tool for long time in-vivo intravital imaging ${ }^{2}$.

While many commercialized and customized two-photon microscopes are available and been used in imaging ${ }^{3}$, we optimize the customized two-photon microscope system to high speed type, for high temporal resolution in-vivo animal studies. With a full utilization of polygon mirror scanning schematic ${ }^{4,5}$, our system can achieve $512 \times 512$ full frame from $5 \mathrm{~Hz}$ to $50 \mathrm{~Hz}$. And with different scanning area and image size, out plant has the ability to rise from $20 \mathrm{~Hz}$ to $1600 \mathrm{~Hz}$ in frame rate and 1024 x 1024 to 16 x 512 in image size. This plant is fully controlled by a self-written program called PScan1.0. People can choose the proper frame rate and resolution to match the fluorescence protein sensors. And also, image digital stabilization process is implemented on our system, which is quite important in in-vivo imaging.

Speed is being concerned as the majority drawback at the very first beginning of two photon laser scanning microscope. With the utilization of resonant scanning mirror, the video rate two photon microscope is realized. However, compare with polygon mirror, which has a dynamic rotation speed range rather than a single resonant frequency, resonant scanning speed is fixed at the resonant frequency. In addition, dynamic rotation speed supplies the ability to control exposure time, which is convenient for matching between spatial and temporal resolution.

*steve.lee@anu.edu.au

SPIE BioPhotonics Australasia, edited by Mark R. Hutchinson, Ewa M. Goldys, Proc. of SPIE Vol. 10013, 100130K · @ 2016 SPIE · CCC code: 0277-786X/16/\$18 · doi: 10.1117/12.2242951 


\section{METHODS}

\subsection{Hardware}

Figure 1 shows the optical part of our system, in which polygon mirror is the fast axis scanning, which combined with the galvo mirrors as the slow axis constituting the scanning mechanism. The planes of all the scanning mirrors are conjugated to the back focal plane of objective. Dichroic beam splitters are used to obtain two color channels, red and green.

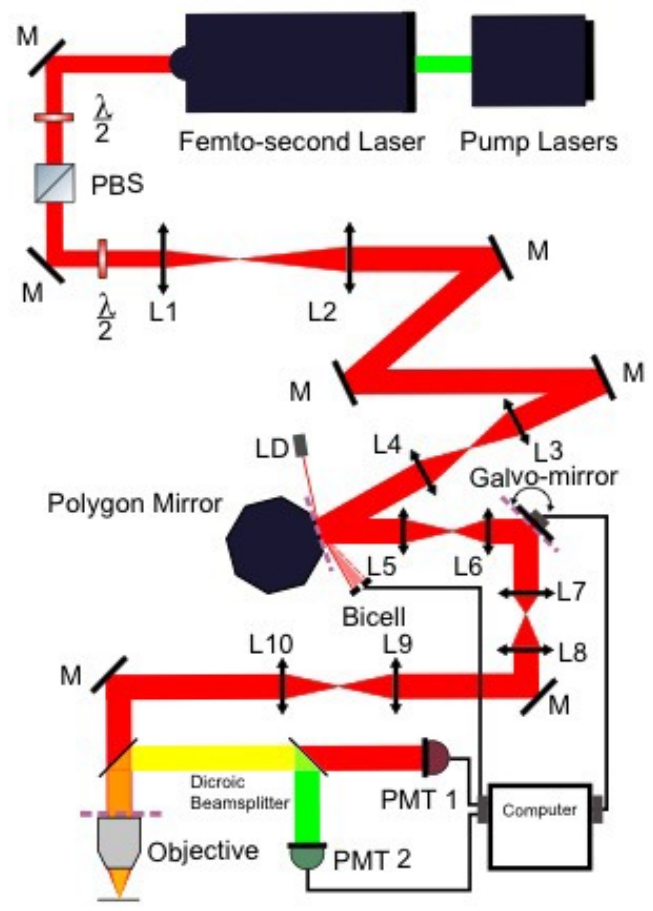

Figure 1. Schematic picture of optics in the polygon mirror microscope.

Figure 2 is the control part of our microscope. A PCI card from National Instruments is utilized to generate the control signal to the microscope drivers. Two analog outputs to the galvo mirror, two counter signals to the drive the polygon mirror and be as the HSYNC for the frame grabber. All the signals are synchronized by the LabVIEW program. 


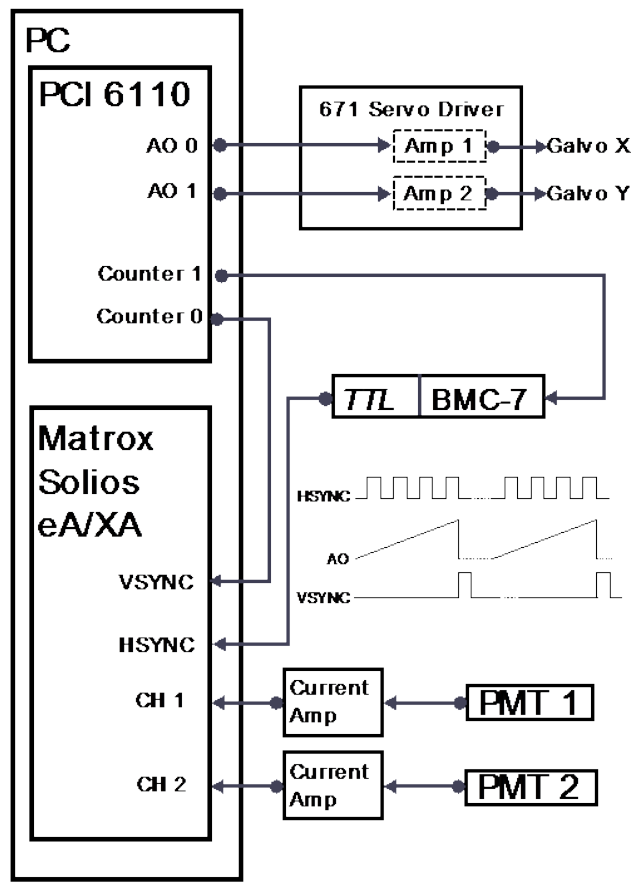

Figure 2. Schematic picture of the microscope controlling.

\subsection{Software}

The control software PScan 1.0 GUI is shown in figure 3. It is a customized self-written software, for filling the gap between polygon mirror control and microscope image acquisition. It has a various interface among script language, ImageJ and LabVIEW.

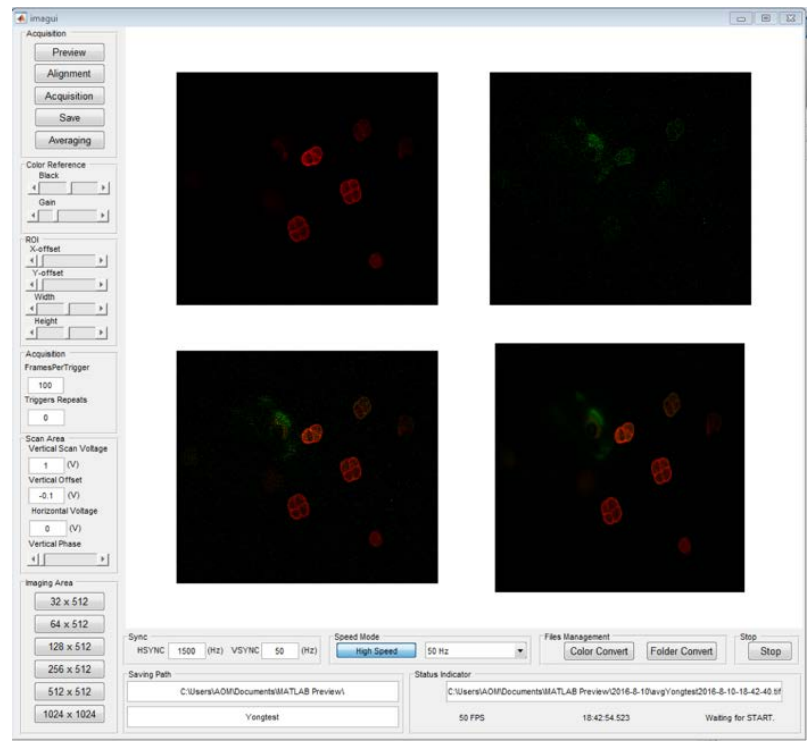

Figure 3. GUI of PScan 1.0. 


\section{RESULTS}

For tissue imaging, we use a thin slice of tissue from brain of Wistar rats. The neurons were dissected from hippocampal region of the brain of neonatal wistar rats, cultured on glass coverslips and the recording was done after 10 days in culture. The fluorescence of Cal-520 significantly increases when the release of intracellular calcium occurs. The imaging results are shown in figure 4, where the $160 \mathrm{~Hz}$ frame rate make more transients observable.

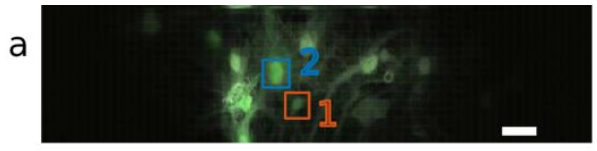

b

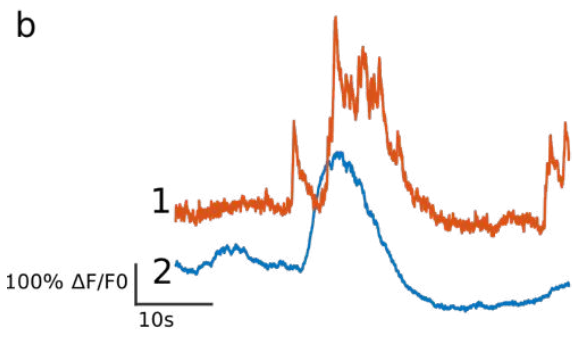

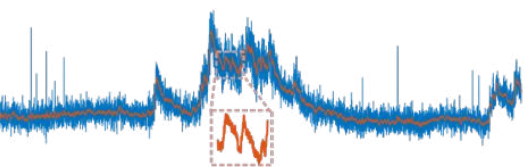

$160 \mathrm{~Hz}$

Figure 4. Imaging experiment of neurons. (a) Standard deviation image of the Wistar rat neuron cell. Scale bar is 50 um. (b)

The signal time trace observed from the image frame stream (a). The time is $52 \mathrm{~s}$ long. (c) The compare between $160 \mathrm{~Hz}$ frame rate acquisition and the corresponding $40 \mathrm{~Hz}$ down sampling image. More transients are observed in the $160 \mathrm{~Hz}$.

For intravital imaging, record of red blood cell flowing is shown in figure.5. The microvasculature of a wild type mouse is highlighted with fluorescent dextran-rhodamine dye. One of the flowing cell is tracked.

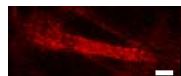
$a^{0.25 s}$
$0.75 \mathrm{~s}$
$1.25 \mathrm{~s}$
$1.75 \mathrm{~s} \quad 2.25 \mathrm{~s}$
$2.75 \mathrm{~s}$
$3.25 \mathrm{~s}$
$3.75 \mathrm{~s}$

b

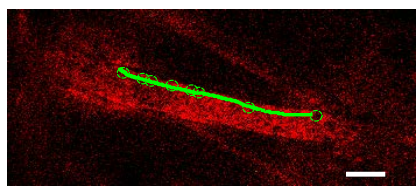

C

Figure 5. In vivo imaging of mouse's micro vasculature. (a) Time sequence image of the vasculature. (b) The trace of the red blood cell flowing. (c) The plot of red blood cell flowing distance over time.

\section{CONCLUSION}

Polygon mirror based two photon microscope is a suitable for high dynamic recording biology experiments. It can capture the events from $5 \mathrm{~Hz}$ to $1600 \mathrm{~Hz}$ dynamically by controlling the rotation speed, which is going to be widely used in the following specific experiments of developmental biology and neuron science. In the next, we will add more imaging modes to expand its functionality. 


\section{REFERENCES}

[1] Denk, W., Strickler, J. H. and Webb, W. W., "Two-photon laser scanning fluorescence microscopy," Science 248(4951), 73-76 (1990).

[2] Myunghwan, C., Woei M. L. and Seok, H. Y., "Intravital Microscopic Interrogation of Peripheral Taste Sensation, " Scientific Reports 5(8661), 1-6 (2015).

[3] Rosenegger, D. G., Tran, C. H. T., LeDue, J., Zhou, N. and Gordon, G. R., "A High Performance, Cost-Effective, Open-Source Microscope for Scanning Two-Photon Microscopy that Is Modular and Readily Adaptable,” PLoS ONE 9(10), 1-16 (2014).

[4] Israel, V., Joel, A. S., David, P. B., Daniel, C. and Charles, P. L., "In Vivo Cell Tracking with Video Rate Multimodality, " IEEE Journal of Selected Topics in Quantum Electronics 14(1), 10-18 (2008).

[5] Ki, H. K., Christof B and Peter T. C. S., "High-speed, two-photon scanning microscope," Applied Optics 38(28), 6004-6009 (1999). 